\title{
LENGUA MINORIZADA Y MEDIOS DE COMUNICACIÓN: PREJUICIO, ACTIVISMO Y FRAGMENTACIÓN LINGÜÍSTICA. EL CASO DEL ARAGONÉS
}

\author{
Mass Media and Minoritized Language: Prejudice, Activism and \\ Linguistic Fragmentation. The Case of Aragonese Language
}

\author{
Iris Orosia CAMPOS BANDRÉS ${ }^{1}$ \\ Universidad de Zaragoza
}

\section{Resumen}

A pesar de que la diversidad lingüística de Aragón ha sido institucionalmente reconocida desde la aprobación de su Estatuto de Autonomía en 1982, la presencia del aragonés en los medios de comunicación autonómicos ha sido escasa y cuestionada. Ante tal situación, esta labor ha sido asumida por activistas pertenecientes principalmente al sector neohablante. Con el fin de realizar una aportación sobre el estado de la cuestión respecto al aragonés en los mass media, se desarrolló un trabajo de naturaleza cualitativa cuyos resultados muestran una actitud mayoritariamente favorable hacia su presencia en los medios, especialmente en los territorios donde todavía se conserva con cierto grado de vitalidad; lugares donde, sin embargo, no tienen repercusión las iniciativas desarrolladas desde el ámbito neohablante, lo cual pone de manifiesto la fractura existente entre la comunidad nativa y los nuevos hablantes de aragonés. Por otra parte, destaca la carencia de información sobre la lengua propia entre la sociedad altoaragonesa. De este modo, en muchas ocasiones emerge una identificación del aragonés con modelos tintados por el prejuicio, tópico y/o folklorismo como son las identidades baturras, difundidas de un modo recurrente a través de los medios de comunicación y del cine, sobre todo a lo largo del siglo Xx.

Palabras clave: lengua aragonesa; actitudes lingüísticas; prejuicios lingüísticos; lenguas minorizadas

1 Departamento de Didáctica de las Lenguas y de las Ciencias Humanas y Sociales de la Universidad de Zaragoza. Correo electrónico: icamposb@unizar.es. Fecha de recepción: 20 de febrero de 2018. Fecha de aceptación: 8 de mayo de 2018. Versión final: 18 de mayo de 2018. 


\section{Abstract}

Linguistic diversity in Aragon has been institutionally recognized since 1982. Nevertheless, its presence in the media has been poor and questioned. Given that situation, this concern has been tackled by activists from the 'new speakers' sphere. With the aim of making a contribution with respect to the Aragonese language in the mass media, a qualitative research was conducted in Alto Aragón. Results show the existence of a majoriatian favorable attitude towards the presence of Aragonese in the media, particularly in the areas where the language has still some level of vitality. Nevertheless, initiatives developed by activists have a very little impact around native speakers of Aragonese, and this situation underscores the gap that exists between 'native speakers' and 'new speakers'. Finally, it is also important to stress the lack of information about the regional language among the population. Thus, it is common to find the identification of Aragonese language with linguistic models tainted with prejudices. One recurring example is the 'baturra' identity, that has been largely widespread by films and the media during the $20^{\text {th }}$ century.

Key words: aragonese language; lingüístic attitudes; lingüístic prejudices; minoritized languages

\section{INTRODUCCIÓN}

El aragonés es una de las dos lenguas minoritarias y minorizadas de Aragón, así como una de las lenguas en mayor riesgo de desaparición de Europa Occidental (Moseley, 2010). Tras una primera etapa de apogeo y esplendor de esta lengua románica, la firma del Compromiso de Caspe en 1412 supuso, con la entrada de la dinastía castellana de los Trastámara en el territorio de la antigua Corona, el inicio de su retroceso. Desde entonces y a lo largo de los siglos, su territorio de uso predominante ha ido menguando y en nuestros días la lengua se encuentra arrinconada en los altos valles pirenaicos y algunos puntos del Prepirineo aragonés, con la excepción de la comunidad de neohablantes, que desde el inicio del movimiento activista por la recuperación de la lengua, en 1970, se ha extendido en algunos núcleos urbanos principalmente de las provincias de Huesca y Zaragoza.

En cuanto al número de hablantes de aragonés, a falta de un censo lingüístico oficial, los datos con los que contamos son escasos y deben interpretarse con cierta cautela. En primer lugar, los datos recopilados a través de una pregunta incorporada al censo oficial de habitantes de Aragón en 1981, presentaban la existencia de 11.824 bablantes activos de aragonés (localizados principalmente en la provincia de Huesca), y de $17.653 \mathrm{ha}$ blantes pasivos, término que hace referencia a las personas que comprenden la lengua pero no son capaces de utilizarla para comunicarse (Nagore, 


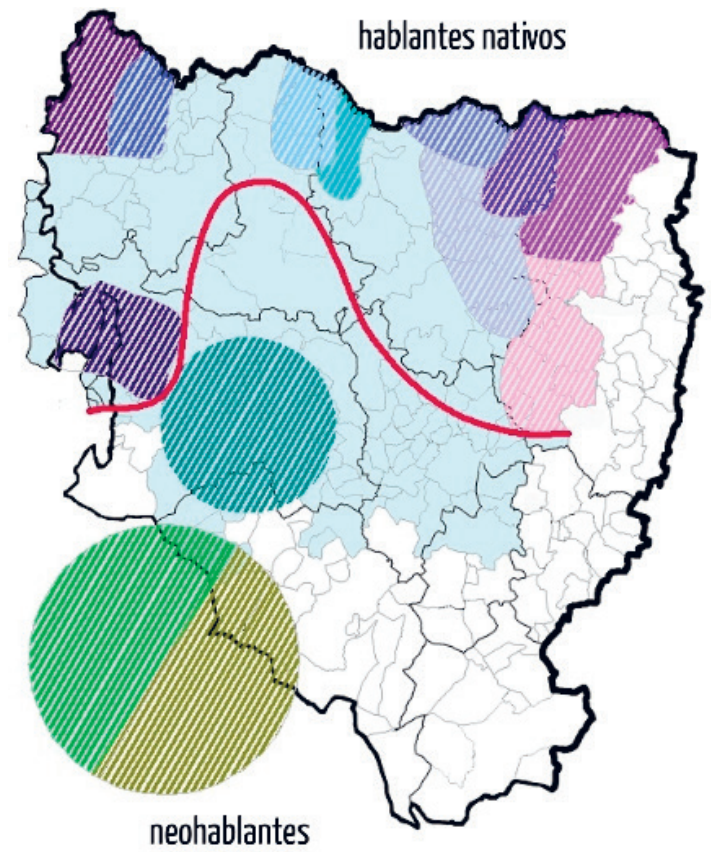

Figura 1. Mapa de la identidad lingüística (fragmentada) de los hablantes de aragonés. Fuente: Campos (2017).

2008). Por otra parte, según un estudio realizado en 2001 en el territorio del Alto Aragón (provincia de Huesca) por el equipo de Euskobarómetro, se determinó que en la provincia de Huesca un total de 24.172 personas entendían y hablaban aragonés, 34.830 lo entendían pero no lo hablaban y 70.570 ni lo entendían ni lo hablaban (Llera, 2001). Los datos más actuales son los recopilados a través del censo oficial de habitantes de Aragón de 2011, según los cuales, en nuestros días, 25.556 personas hablan aragonés (1,9\% de los habitantes de la Comunidad), y el número de gente que lo conoce aumenta hasta los 56.235 aragoneses (4,2\% de los habitantes de la Comunidad). También según esta fuente, resulta importante señalar que un tercio de las personas que conocen el aragonés hoy se encuentra en la provincia de Zaragoza, lo cual podría significar el incremento de la comunidad neohablante.

En cuanto a la distribución y cohesión de los hablantes de aragonés, de nuevo contamos con datos parciales pero, en síntesis, se puede considerar 
que, al igual que señala Alén (2013) para el caso del occitano en Francia, en nuestros días no podemos hablar de la existencia de una comunidad lingüística entendida en los términos de Labov, sino más bien de la presencia de varias redes de hablantes dispersas en diferentes puntos del territorio aragonés y, además, fragmentadas desde el punto de vista sociolingüístico (Figura 1). De este modo, estudios como los de Postlep (2012) o Campos (2017) han constatado la presencia de un discurso, entre los hablantes nativos de aragonés, marcado por la negación de su pertenencia a una comunidad lingüística aragonesohablante homogénea, entendida como el conjunto de hablantes nativos de todas las variedades dialectales de la lengua y los neohablantes localizados en ámbitos urbanos como Huesca y/o Zaragoza.

En la Figura 2, sobre el territorio de influencia histórica de la lengua aragonesa (López, 2012), aparecen en los dos tonos más oscuros los lugares donde el aragonés todavía es conocido por algunas personas mayores (tono intermedio) y utilizado con un cierto grado de bilingüismo social aunque en fuerte estado de diglosia (tono oscuro). Estas dos zonas incluyen los valles de Ansó, Echo, Bielsa, Chistau y Benás, la zona sur-este de la Ribagorza y algunas localidades de la Galliguera y el Valle de Tena. A ellas, como ya se ha señalado, hay que sumar el considerable número de personas neohablantes de aragonés que se encuentran, fundamentalmente, en la capital oscense y en la provincia de Zaragoza.

En lo que respecta a la protección de la lengua aragonesa desde las instituciones públicas, ésta, ha estado marcada por el abandono hasta bien entrado el siglo Xx. Uno de los hitos en cuanto a la implicación de agentes institucionales en pro de la salvaguarda de la lengua lo encontramos en la denuncia que Emilio Gastón, entonces Justicia de Aragón, realizó en 1993 a través de su Informe sobre las lenguas minoritarias en Aragón (Justicia de Aragón, 1993). Gastón dedicó un informe exclusivamente a la cuestión lingüística en Aragón, el cual, tal y como apunta Garcés (2015), respondía a «la avalancha de quejas demandando la cooficialidad del aragonés y del catalán junto con el castellano» (Garcés, 2015: 186). En este texto, el entonces Justicia de Aragón subrayaba la necesidad de otorgar un reconocimiento jurídico a la realidad plurilingüe de la Comunidad. Esta acción de denuncia de la situación de la lengua aragonesa, unida a las reivindicaciones realizadas por las asociaciones de hablantes y a los informes emitidos por organismos internacionales como la UNESCO (1996), han derivado en las tres últimas décadas en el desarrollo de algunas acciones desde el plano político-administrativo como fue, en 1997, la aprobación en las Cortes de Aragón del Dictamen de la Comisión especial de política lingüística 


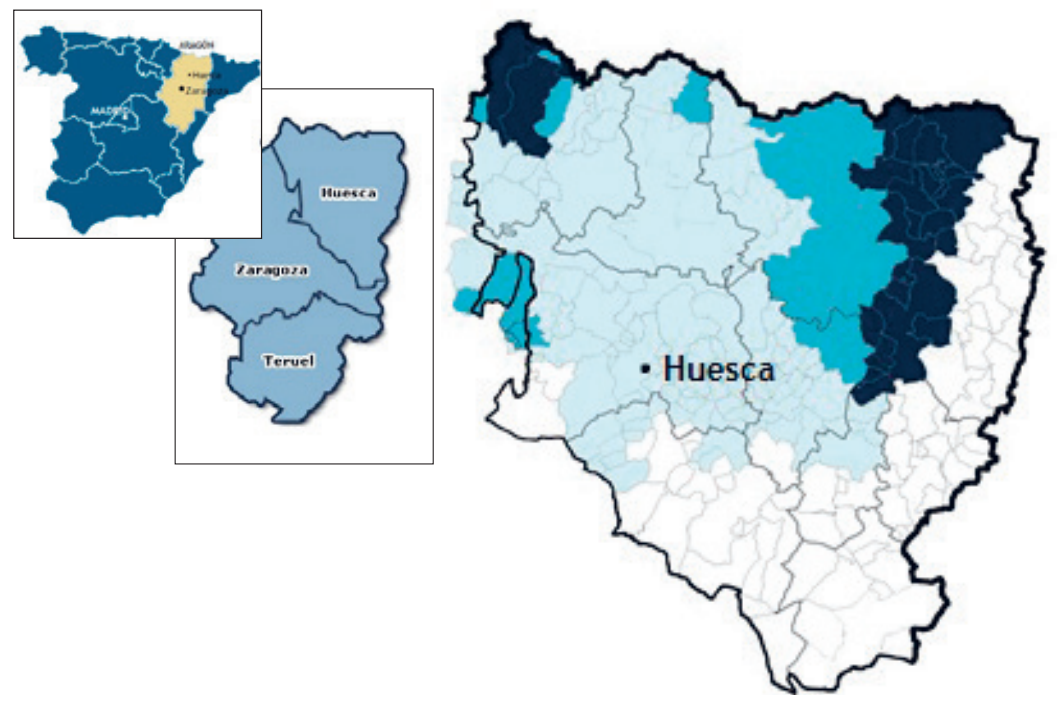

Figura 2. Territorios y grado de vitalidad de la lengua aragonesa. (Elaboración propia a partir de Postlep, 2012).

(Cortes de Aragón, 1997), donde se esbozaban las directrices a seguir para la creación de una Ley de Lenguas de Aragón y se animaba a su aprobación antes de terminar ese mismo año. A este dictamen le siguieron en 1998 un borrador de Anteproyecto de la Ley de Lenguas de Aragón que no fue llevado a las Cortes y en 1999 la aprobación de la Ley de Patrimonio Cultural de Aragón, donde se manifestaba nuevamente la necesidad de aprobar una ley de lenguas de Aragón y se reconocía por primera vez la realidad trilingüe de la Comunidad mediante la denominación de cada una de sus lenguas minoritarias bajo su glotónimo científico: aragonés y catalán. Hasta entonces, la ambigüedad respecto a la cuestión lingüística en el plano político se hacía patente a través del texto del Estatuto de Autonomía aprobado en 1982, que en su Artículo 7 recogía que: «las diversas modalidades lingüísticas de Aragón gozarán de protección, como elementos integrantes de su patrimonio cultural e histórico» (Cortes de Aragón, 1982: 22033), sin concretar qué se entendía bajo el concepto de «modalidades lingüísticas» y sin poner nombre a las lenguas de Aragón.

En el año 2001, se publicó un nuevo anteproyecto de Ley de Lenguas que no derivó en la presentación de un proyecto de ley, postergándose la aprobación de un marco legislativo para las lenguas de Aragón hasta el año 
2009 (López y Soro, 2010), bajo la Ley 10/2009, de 22 de diciembre, de uso, protección y promoción de las lenguas propias de Aragón, la cual, sin embargo, no llegó a aplicarse. La escasa efectividad institucional en lo que respecta a la dignificación y salvaguarda del patrimonio lingüístico aragonés se corroboró nuevamente tras el cambio del signo político en el Gobierno de Aragón en 2011, lo cual supuso la derogación de la Ley de Lenguas de Aragón de 2009 y la aprobación de un nuevo texto en 2013, el cual establecía más limitaciones al desarrollo de las lenguas propias, además de generar una controversia de considerable alcance al privar a estas lenguas de sus glotónimos científicos, aceptados desde el ámbito de la Romanística a nivel internacional, y acuñar, en cambio, unas nuevas denominaciones para las mismas: Lengua aragonesa propia de las áreas pirenaica y prepirenaica (conocida de forma extraoficial y ajena al texto legislativo bajo las siglas $L A P A P Y P$ ) para el caso del aragonés, y Lengua aragonesa propia del área oriental (conocida de forma extraoficial y ajena al texto legislativo bajo las siglas $L A P A O$ ) para el caso del catalán de Aragón (López Susín, 2015).

Tras dos años de aprobación sin aplicación del texto legal, un nuevo cambio en el signo político del Gobierno de Aragón trajo consigo en 2015 el inicio de una nueva etapa en lo que respecta a la realidad lingüística de la Comunidad. Así, en dicho año se creó la primera Dirección General de Política Lingüística del Gobierno de Aragón, institución desde la cual se están realizando labores de promoción y normalización lingüística mediante el comienzo de aplicación del texto legislativo de 2013.

\subsection{Los medios en aragonés: activismo y resistencia ${ }^{2}$}

El papel que ha jugado el aragonés en los medios de comunicación desde comienzos del siglo Xx ha sido limitado a consecuencia de la falta de protección otorgada desde el ámbito institucional y su consecuente estado de diglosia, con un uso de la lengua relegado principalmente al ámbito rural y a situaciones cotidianas o familiares. De todas formas, se pueden diferenciar dos etapas: una inicial desde inicios del siglo hasta la década de 1970, cuando comenzó el proceso de «Renaxedura» del aragonés (López, 2012b); y una segunda, desde entonces y hasta el final de siglo, que se ha extendido hasta nuestros días.

2 Para mayor información, consultar Eito (2013) y Ramos (2014). 
En la primera época, el papel que tenía el aragonés en los medios queda reflejado en las colaboraciones de una nómina de escritores en revistas y publicaciones periódicas del Alto Aragón, fundamentalmente locales. Así, autores como Marcelino Gambón, Veremundo Méndez, Agliberto Garcés, Dámaso Carrera, Cleto Torrodellas o Leonardo Escalona realizaban aportaciones en publicaciones como el Heraldo Ribagorzano o La Tierra, pero todas ellas con un objetivo de difusión artística, es decir, sus publicaciones no tenían una función informativa sino que estos autores buscaban la difusión de su obra literaria a través de tales medios. De este modo, la presencia del aragonés como vehículo de transmisión de información, opinión, etc., era muy limitada ya a principios del siglo XX. Un curioso y excepcional ejemplo es un texto publicado en 1934 en el Periódico La Tierra en formato epistolar y que denunciaba la incomunicación sufrida por los habitantes del valle oscense de Nozito.

También destacaron en esta primera etapa las aportaciones de los aragoneses emigrados a Barcelona, a través del boletín del Centro Aragonés de Barcelona, publicado desde 1909, con las colaboraciones de Privato Cajal, entre otros. Del mismo modo, la revista aragonesista El Ebro contó entre 1917 y 1936 con las aportaciones de autores que reivindicaron «la existencia del aragonés como realidad cultural pero no sociocultural» (Serrano, 2014: 10), vinculada a la tradición y al folklore, reflejándose así la realidad de una lengua en estado de diglosia. De este modo, los textos que aparecían también en esta publicación durante las primeras décadas del siglo XX tenían una naturaleza alejada de la intención informativa; siendo o bien literaria, folklórica o costumbrista, o bien científica o pseudocientífica, a través de trabajos de recopilación y estudio lingüístico (Serrano, 2014).

Ya en la década de 1940, el aragonés comenzó a tener su espacio en publicaciones de considerable calado como el Heraldo de Aragón, diario en el que el humanista altoaragonés Pedro Arnal Cavero colaboró de forma habitual en lengua aragonesa (Gracia, 2014), también con una finalidad principalmente de difusión literaria.

Con el nacimiento del movimiento activista vinculado a la salvaguarda del aragonés, en la década de 1970, surgió una segunda etapa respecto a la presencia de la lengua en los medios de comunicación. Desde el movimiento activista se contemplaba como acción prioritaria la difusión de la lengua entre la población altoaragonesa, para lo cual algunos círculos comenzaron a ayudarse de los medios de comunicación en la medida de sus posibilidades. Esto derivó en la paulatina aparición de nuevas publicaciones que 
se sumaron a otras, de alcance local y comarcal, en las que las variedades del aragonés utilizadas por sus hablantes nativos tenían cabida, aunque desde una perspectiva diglósica similar a la de las publicaciones de la primera etapa del siglo XX. Así, mientras la revista Fuellas, vinculada al Consello d'a Fabla Aragonesa (Huesca), nacía en 1978 con un espíritu de reivindicación y difusión de información en lengua aragonesa, al mismo tiempo se consolidaban publicaciones como Jacetania, revista comarcal que entre sus artículos en castellano difundía desde de 1966 la obra literaria en aragonés de autores como Veremundo Méndez o Rosario Ustáriz, colándose, únicamente, entre los poemas, relatos y chascarrillos, los escritos reivindicativos de neohablantes activistas como Francho Nagore o Chusé Aragüés. Esta realidad muestra la dicotomía en la que se comenzaba a encontrar el aragonés en los albores de la democracia y que, como muestra Campos (2017), parece haberse extendido hasta nuestros días. Una dicotomía en la que encontramos dos realidades de hablantes: la de los nativos, vinculados a un empleo diglósico del aragonés, y la de los neohablantes, vinculados a un movimiento de resistencia que lucha por llevar la lengua a todos los ámbitos de la vida del siglo XXI.

Fruto del afán normalizador fueron el nacimiento, en la década de 1980, de la revista L'orache, vinculada al Ligallo de Fablans de L'aragonés (Zaragoza), y, ya en 1990, el de la revista de miscelánea O Espiello, vinculada a la asociación cultural Nogará (Zaragoza). Otras publicaciones vinculadas al aragonesismo también dieron y dan acogida a aportaciones de todo tipo escritas en lengua aragonesa, como es el caso de Siete de Aragón, Rolde u O Rayón. Asimismo, cabe destacar que revistas de alcance local como El Alcabué o Bisas de lo Subordán han supuesto lo que parece ser una pequeña muestra de cierta ruptura con el uso folklórico y literario de la lengua propia en los medios de comunicación por parte de los hablantes nativos.

Si dirigimos la atención a las muestras más recientes de uso del aragonés en los medios, cabe hacer alusión a su llegada a los ámbitos radiofónico y digital, gracias al auge y socialización de la Internet. De este modo, en las dos últimas décadas han nacido numerosas iniciativas, todas ellas vinculadas al ámbito activista y neohablante, como el programa semanal de radio Fendo Orella (en la emisora libre Radio Topo, Zaragoza; desde 2004) o los ya desaparecidos Charramos, de Radio Huesca (desde 1980 hasta 2001, vinculado al Consello d'a Fabla Aragonesa), o Charradetas y Capazos, de Radio Somontano. El pasado año, el nacimiento de la emisoria online Chisla Radio, con la ayuda de la Dirección General de Política Lingüística del Gobierno de Aragón, supuso un nuevo paso hacia adelante. Asimismo, 
iniciativas como el periódico online Arredol (desde 2011) o el canal de televisión digital recientemente aparecido, Ueito $T V$, amplían el elenco de medios que ofrecen un uso normalizado del aragonés en su labor informativa. A ellos se suman otras iniciativas de tipo amateur con cierto alcance y aspiración comunicativa como son el canal de cocina alojado en Youtube A Escuchetes o los blogs y páginas web de las diferentes asociaciones que actualmente trabajan por la salvaguarda del aragonés.

Para finalizar, debemos apuntar que estas iniciativas conviven hoy con el todavía vigente empleo del aragonés para difundir textos de creación literaria de tipo costumbrista, que ofrecen una visión diglósica de la lengua como medio de expresión idóneo para el retrato de una sociedad rural ya extinta. Un ejemplo paradigmático es la publicación semanal en el Heraldo de Aragón, el periódico de mayor tirada de la Comunidad, de la sección «Carasol aragonés», donde se pueden leer textos como el que sigue:

Nomás quedaba tres casas ubiertas en o lugar, tres chamineras que fumiaban. Antonet de Casa Nabarro nos viyeba trachinar dende a suya portera, rodiau por as fumatas d'a suya pipa, que no le deixaban alentar como yera menister. Pareixeba que no parava cuenta d'o que pasaba, pero me creigo que no las teneba todas, pos puyaba todas as nueitz a pasar a veilada con nusotros y, de paso, revisalsiar a nuestra casa. 'Serafina, ista concina no está como dinantes, faltan os calders, os cantaros, os banquetz, os embotidos que s'ixoriaban en ixos palos...' [...] Cuan se fizo la hora de marchar, Antonet se cantó d'a cadiera y yo salié con o candil pa amostrar-le as escaleres [...] Ixas fuen as zagueras parolas que le sintié en a nuestra casa, parolas que se quedón sin respuesta, pos cuasi se me gastó a gana, cuan vide que se sacaba o moquero d'a pocha, pa enchugar-se as glarimas en o patio. Ixa estampa d'hombre acotolau, se me fincó pa siempre en o mío peito. (Satué, 2016)

\subsection{El aragonés en los medios: censura y prejuicio}

De forma paralela a la aparición y desarrollo de los medios de comunicación en aragonés, consideramos necesario hacer un repaso a la imagen del «aragonés-tipo» difundido ad nauseam a través de este tipo de medios de comunicación a nivel estatal y que puede haber tenido influjo en las representaciones de la población respecto a la lengua propia; así como repasar, también, el papel que la administración de la Comunidad ha tenido y tiene en cuanto al desarrollo de esta lengua en el área de los medios de comunicación autonómicos.

El nacimiento, en los albores del siglo XXI, de una corporación de radio y televisión autonómica supuso un momento de esperanza desde el ámbito 
del activismo lingüístico vinculado a las lenguas propias de Aragón. Sin embargo, tras más de una década en funcionamiento, la presencia del aragonés en estos medios es prácticamente nula y se reduce a un tratamiento parcial en algunos magazines o programas de humor y de entretenimiento como Bien Dicho u Oregón Televisión. Esta realidad es muestra del incumplimiento tanto la ley de lenguas aprobada en 2009 como de la actualmente en vigor. Así, el texto de 2009 rezaba en su Artículo 35, el cual es literalmente similar al Artículo 24 de la ley de 2013:

Artículo 25. Medios de comunicación. Respetando los principios de independencia y de autonomia de los medios de comunicación, las Administraciones Públicas adoptaran las medidas adecuadas a fin de alcanzar los siguientes objetivos: a) Promover la emisión en las radios y televisiones públicas de programas en las lenguas propias de Aragón de manera regular; b) Fomentar la producción y la difusión de obras de audición y audiovisión en las lenguas propias; c) Fomentar la publicación de artículos de prensa en las lenguas propias de manera regular; d) Ampliar las medidas existentes de asistencia financera a las produccions audiovisuales en lenguas propias; e) Apoyar la formación de periodistes y demás personal para los medios de comunicación que empleen las lenguas propias y f) Velar para que los intereses de los hablantes de lenguas propias estén representados o sean tomados en consideración en el marco de las estructures que se puedan crear, de conformidad con la ley, con el objeto de garantizar la libertad y la pluralidad de los medios de comunicación. (López y Soro, 2010: 125)

En cambio, lo que sí se ha difundido a través de los principales medios de comunicación tanto autonómicos como estatales durante décadas es la imagen estereotipada de los aragoneses desarrollada desde mediados del siglo XIX en algunos círculos del ámbito artístico, literario y periodístico con Zaragoza y Madrid como sus principales focos de difusión. Así, tal y como señala García:

Hay que dejar sentado que la imagen de Aragón se proyectó fundamentalmente desde Madrid, promovida por aragoneses e impulsada desde periódicos, revistas y otras ediciones de formato menor, muchas veces con exceso de ganga o chascarrillos o, en el peor de bastantes casos, chistes palurdos. (García, 1999: 115)

Desde entonces, a través de los medios de comunicación y el cine ha sido recurrente la identificación del habitante de Aragón con la del baturro, imagen que algunos autores consideran: «la primera falsificación de lo aragonés, la primera mixtificación de las maneras y el habla de un pueblo campesino henchido de virtudes, auspiciada por unos cuantos señoritos pastosos que pugnaban por distanciarse de su pueblo, del que sentían ver- 
güenza» (Guillén, 2012: 1), y cuyo objetivo central era la aculturación mediante el humor. De este modo, autores como Guillén (2012) destacan el origen de esta identificación como el resultado del dominio de lo urbano sobre lo rural, de la burguesía sobre el pueblo, respondiendo a un proceso de dominio mediante el ejercicio de la violencia simbólica en términos de Bourdieu (2008). Como consecuencia de ello, en la actualidad, una parte de la población aragonesa todavía disfruta viéndose reflejada en los personajes de Paco Martínez Soria o Marianico «El Corto», y esta realidad explica el éxito de humoristas como Miguel Nadal («Miki Nadal»). En palabras de Guillén (2012: 1), en nuestros días

quienes rechazan verse burlonamente reflejados en una caricatura baturra que no reconocen como suya - porque la miran desde arriba o desde fuera- conviven con los sectores populares aragoneses que, lejos de interiorizar este tono despectivo, se sienten orgullosos de verse y reconocerse en espacios tan mitificados como eran entonces el periodismo y la literatura o después han sido el cine y la televisión [...] muchos aragoneses viven hoy esa representación distorsionada de sí mismos al margen del gusto culto y hasta de su espesor ideológico. Simplemente disfrutan viéndose en espacios públicos de poder, esos que ellos consideran más importantes y más suyos.

En lo que respecta a la cuestión lingüística, consideramos que este estereotipo de la identidad aragonesa ha llegado hasta nuestros días con las mismas repercusiones para la lengua que tuvo en sus orígenes. En este sentido, Enguita apunta que, con el nacimiento del tópico del baturro, la forma de hablar «rústica» en Aragón, la del ámbito rural, una lengua con mayor o menor presencia de elementos léxicos y gramaticales del aragonés, se convirtió en el medio de expresión de ese personaje zafio, grotesco, bruto, dibujado por autores como Alberto Casañal, Sixto Celorrio o Mariano Baselga (Enguita, 2014: 145). Así, tal y como destaca Ciprés (2009: 13): «ya a finales del siglo XIX, como consecuencia de las ideas románticas ligadas al popularismo y al costumbrismo, se empezaron a utilizar expresiones aragonesas insertas en un discurso castellano como marcas de identidad aragonesa, hecho que incurría en el denominado baturrismo.»

La identificación y expansión de estas expresiones, y en última instancia del hablante de aragonés, con esos personajes estereotipados habría estado íntimamente ligada a la realidad de las élites intelectuales del Aragón de la época, entre las cuales predominaban el desconocimiento, la confusión, e incluso la ocultación de la realidad plurilingüe del territorio aragonés. Así, tal y como destaca Aliaga (2012), «la intelectualidad aragonesa 
desconocía profundamente la diversidad lingüística de Aragón y andaba lejos de reconocer que en el territorio aragonés convivían tres lenguas románicas diferenciadas» (Aliaga, 2012: 27), hecho agravado por «la inexistencia en la universidad zaragozana de profesionales de la lingüística de las lenguas vivas vinculados, siquiera en alguna medida, con la pujante romanística centroeuropea de la época» (Aliaga, 2012: 27).

Por otra parte, tal y como señala el mismo autor, la lealtad política de estas élites a una España cohesionada, dejaba a un lado cualquier aspiración de instrumentalización simbólica de una lengua propia diferente al castellano, por lo que la «diferencia en la unidad» se mostraba como el camino lógico para el tratamiento de la realidad lingüística aragonesa si se tienen en cuenta las características sociopolíticas de la época. De esta manera, en los albores del siglo XX, las élites intelectuales aragonesas (mayoritariamente de procedencia zaragozana) centraron sus esfuerzos en la puesta en valor de un castellano regional, aragonesizado, que encajaba, al mismo tiempo, con sus ideales políticos y con la exaltación de la diferencia propia del romanticismo de la época.

Tal y como recordábamos anteriormente a través de Enguita (2014), Aliaga también recuerda que «la novela costumbrista, las piezas teatrales tragicómicas y los cantares acogieron la (re)creación del habla regional» y que «a algunos literatos se les fue de las manos la inspiración artística y terminaron forjando un estereotipo negativo del aragonés y de su lenguaje que perdura en la actualidad, si bien no faltaron voces críticas que se alzaron contra la deriva ridiculizante que habían adoptado estas expresiones literarias» (Aliaga, 2012: 28).

La realidad de ambigüedad e ignorancia, en términos lingüísticos, que marcaron las décadas finales del siglo XIX y las primeras del XX, consolidada y difundida a lo largo del tiempo, habría ayudado a asentar y permitir la llegada hasta nuestros días de una serie de tópicos sobre la lengua aragonesa y sus hablantes.

\section{Diseño De NUESTRA INVESTIGACión}

\subsection{Objetivos de investigación}

El propósito de la investigación desarrollada es fundamentalmente exploratorio y descriptivo, con la intención de aportar datos sobre una realidad apenas estudiada desde la perspectiva sociológica, la del papel del ara- 
gonés en los medios de comunicación. Nuestros objetivos de investigación han sido, concretamente, los siguientes:

a) Explorar la situación de los medios de comunicación en aragonés a través del discurso de la comunidad de activistas neohablantes que han impulsado estas iniciativas.

b) Identificar el grado de repercusión de los medios de comunicación desarrollados en aragonés entre la población altoaragonesa, con especial atención a los hablantes nativos de aragonés, con el fin de aportar un nuevo dato que corrobore o ponga en cuestión la fragmentación de la comunidad aragonesohablante apuntada por Postlep (2012) y Campos (2017).

\subsection{Metodología}

Nuestro corpus de datos tuvo una naturaleza cualitativa. De este modo, las técnicas de nuestro trabajo de campo fueron la entrevista semi-estructurada y el grupo de discusión.

Para el análisis de los datos, de naturaleza narrativa y fruto de la transcripción de todas las grabaciones, se consideró oportuna una orientación basada en la Teoría Fundamentada en los Hechos de Glaser y Strauss (1967). Dentro de este método, que consiste en la relación de los datos para la elaboración de una propuesta explicativa a través de sus puntos comunes, escogimos concretamente la estrategia del método comparativo constante. Esta estrategia implica la recolecta, codificación y análisis de los datos de forma simultánea. Nuestro proceso de análisis contó con varias etapas, que se pueden sintetizar en tres fases principales:

1. La primera fue una fase de descubrimiento en la que se identificaron temas y conceptos y se desarrollaron algunas proposiciones, continuando al mismo tiempo el proceso de recolecta hasta llegar a la saturación, es decir, hasta que de los datos obtenidos en el campo no emergieran nuevas claves explicativas.

2. En la segunda etapa, ya con todos los datos recogidos, se llevó a cabo un trabajo más exhaustivo de codificación, con la obtención de categorías, para alcanzar una mayor comprensión de la realidad objeto de estudio.

3. En la fase final, se buscó el distanciamiento necesario para analizar de nuevo los datos con una mayor perspectiva que permitiera comprenderlos dentro de su contexto pero sin formar parte de este. 
Como se ha señalado, el contenido de las entrevistas (un total de 73 horas de grabación audiovisual) fue transcrito y codificado en temas o categorías que permitieron establecer los hechos más significativos. Para realizar esta labor se contó con la ayuda del programa informático ATLAS.ti, en su versión 6.0.

\subsection{Muestra}

Tras aplicar un muestreo no probabilístico de tipo bola de nieve, en total, contamos con 72 participantes de 26 localidades de la provincia de Huesca (hablantes y no hablantes de aragonés) y, por otra parte, contamos con los testimonios de 10 neohablantes vinculados a proyectos de activismo lingüístico relacionados con los medios de comunicación y procedentes de la capital aragonesa, Zaragoza.

\subsection{Resultados}

\subsubsection{Objetivo 1}

Nuestro primer objetivo era «Explorar la situación de los medios de comunicación en aragonés a través del discurso de la comunidad de activistas neohablantes que han impulsado estas iniciativas».

En los discursos de los activistas que desarrollan medios de comunicación en aragonés emergen tres categorías de forma recurrente.

En primer lugar, destaca la alusión al olvido del aragonés en los medios, así como la denuncia de la censura y la falta de apoyo desde la administración pública a las personas que intentan paliar estas carencias mediante iniciativas privadas:

Cuando se empezó a hacer algo de radio en Aragón y para Aragón, esa radio tuvo un crecimiento muy lento y, por supuesto, la asignatura pendiente, a pesar de que por fin tenemos televisión autonómica, ha sido su desarrollo. Ni la televisión ni la radio autonómica han prestado nunca la atención debida al aragonés [...] Los gobiernos han hecho caso omiso de esta realidad social, de esta realidad cultural que es nuestro propio idioma [...] lo que queda es lo que podamos hacer cada uno de los profesionales a nivel particular, que en general es bastante poco.

Yo creo que el papel que están jugando las administraciones en la promoción del aragonés en los medios de comunicación ha sido prácticamente nulo y, de hecho, muchas veces incluso han puesto obstáculos a la presencia del aragonés. Muchas veces hemos visto..., o sabemos, que gente que intenta hablar en aragonés en televisión o que envían cartas en aragonés a los periódicos, han 
intentado esconder esa presencia del aragonés [...] Se ponen obstáculos a la labor que intentamos hacer, que creo que tiene una demanda social y que debería cumplir el Gobierno o la Administración en general.

Ya que no lo hacen las administraciones, intentamos hacerlo nosotras. ¿Qué pasa?, que nosotras también llegamos a lo que llegamos, tenemos nuestros medios y altavoces pero no son suficientes para revertir la situación de esta lengua, que tiene más hablantes que aquellos a los que podemos hacer llegar nuestra voz.

Vinculado a lo expuesto, encontramos una reiterada denuncia respecto a la difusión de estereotipos de la cultura y lengua aragonesas en los medios de comunicación autonómicos, así como la falta de referencias cultas del hablante de aragonés en los mismos, lo cual tiene consecuencias negativas en la construcción del ideario de la población receptora respecto a los hablantes de aragonés:

[...] hay que poner en valor el aragonés en la sociedad. Salir en los medios de comunicación, en la radio..., políticos hablando en aragonés o, al menos, defendiéndolo; o hacerse parodias como con Rita Barberá, ¡pero debe salir! Tiene que haber un referente, y el referente siempre debe estar ahí, porque si no lo tienes, no tienes una meta, y si no tienes una meta, ¿para qué vas a caminar? [...] Tienes que tener un fin y ese fin son los modelos y los modelos deben ser claros, concisos y muy representativos. Y como no los hay pues no..., ni se le da una visión correcta visualmente. No hay ningún modelo del aragonés [en los medios] ¿Quién puede ser un modelo del aragonés, no para ti ni para mí, sino para alguien que esté por aquí, por este edificio, Marianico 'el Corto'? Nadie te va a decir que un Francho Nagore, Raúl Usón, Pilar Benítez... Te van a decir que Marianico 'el Corto', y la realidad es esa y es así, pues bueno...

Cuando aparece el aragonés en la televisión pública es para relacionarlo con el costumbrismo, las cosas de antaño, el campo, lo rural... Y cuando las asociaciones y el movimiento de defensa del aragonés hacemos algo, en general, una actividad o cualquier cosa, es muy difícil que tenga una difusión en los medios de comunicación autonómicos [...] Muchas veces hemos dicho que se nos ignoraba.

Para finalizar, el discurso de estas personas es un discurso de resistencia; consideran su labor de difusión desde una perspectiva más bien ideológica, como una aportación al movimiento de defensa de la lengua y no como el resultado de una necesidad comunicativa, y encuentran en el aragonés una lengua de batalla:

Nos toca trabajar, difundir la lucha de la gente que aprende y habla aragonés, y de la que vive en aragonés. Ayudando a que se difunda conseguiremos que haya cada vez más gente que atienda a los medios de comunicación en aragonés, que compre en el mercado de la cultura en aragonés... Pero somos frágiles y es posible que, con la sola influencia de unos pocos factores negativos, todo el gran trabajo y el esfuerzo que hacemos y hemos hecho durante 40 años se disipe. 
Yo creo que no nos tenemos que conformar con los pequeños pasos que se han dado. El aragonés es una lengua de batalla, parece que tenemos que pelearnos cada día con la sociedad para convencerles de que hablamos y vivimos en aragonés. Tenemos que superar esta situación y eso pasa por que los medios de comunicación, detrás de los cuales están los políticos y políticas, reconocieran que el aragonés es una lengua viva que se habla y que hay personas que vivimos cada día en esta lengua.

\subsubsection{Objetivo 2}

Nuestro segundo objetivo era «Identificar el grado de repercusión de los medios de comunicación desarrollados en aragonés entre la población altoaragonesa, con especial atención a los hablantes nativos de aragonés, con el fin de aportar un nuevo dato que corrobore o ponga en cuestión la fragmentación de la comunidad aragonesohablante apuntada por Postlep (2012) y Campos (2017)».

Entre la población del territorio de influencia histórica del aragonés (en la provincia de Huesca) encontramos diferentes tipos de discurso, los cuales están determinados principalmente por el tipo de contacto del sujeto con la lengua aragonesa, así como por su procedencia.

En primer lugar, entre los hablantes nativos del aragonés, grupo minoritario, encontramos, para comenzar, el nulo conocimiento de la existencia de las iniciativas desarrolladas desde el ámbito activista neohablante. En cualquier caso, la actitud general es favorable a una hipotética presencia de la lengua propia en los medios de comunicación, aunque existe una dicotomía entre este deseo y el rechazo que muestran de forma mayoritaria hacia una lengua aragonesa estandarizada, que sería la utilizada en los mass media:

Respecto a la promoción de la lengua propia en los medios de comunicación, ¿cómo reaccionaríais vosotros ante un programa en aragonés?

S1: está bien.

S2: estaría bien, jya nos ponen mucha jota! Tanta jota, tanta jota... Pues que pongan el aragonés...

S3: pues me parecería muy bien, porque aquí vienen muchos turistas y viene mucha gente de Zaragoza, de Huesca capital, de Teruel..., y te oyen hablar y te preguntan que si hablamos catalán. Y dices: «¿pero cómo puede ser que una cosa tan de aquí y una cosa tan bonita ique es una lengua propia, tú!, y que no sepan NI QUE EXISTE? Luego ya te podrá gustar más o te podrá gustar menos, ipero es que casi todo el mundo sabe que en Fraga se habla el catalán! Vale. Pero que en Benasque, en Echo, en Chistau..., se habla otra cosa.... 
S3: pero en Echo no se habla patués como se habla aquí, ¿eh? No, no tiene nada que ver... No tiene nada que ver. Yo he estado 4 o 5 veces allí... Y sí, que se hablará...

S1: pero es que aquí nos suena rarísimo... Pero es que es patués pero suena raro, tú lo oyes y suena muy raro...

S2: ¡pero es que el patués de Las Paúles tampoco es el mismo que el de aquí!...

Esa es una de las cosas que os quiero preguntar: ¿vosotros qué pensáis sobre un aragonés estandarizado, una lengua estándar...?

S1: ¿estándar? NO, eso no puede existir, eso es un fracaso... ¡ESO NO PUEDE SER!

S3: a mí no me parece..., a mí no..., cada sitio tiene su forma de denominar sus cosas...

S4: pero puede ser una gramática común y luego que en cada sitio que se hable de una forma, ${ }_{j}$ es que, si no, no sacamos ni una cosa ni otra! ${ }_{i}$ HOMBRE! [...] Y a los críos de Almudévar, ¿si les tienes que dar algo...? Pues aprenderán el oficial y a mí eso no me parece mal.

De este modo, muchos de estos hablantes reaccionan con cierta extrañeza cuando se les presentan documentos audiovisuales en un aragonés común o estándar y muestran su rechazo a identificarse con ese modelo de lengua:

[Tras visionar una emisión en aragonés en televisión]. S70: Mmmm... Eh..., a mí me sonaba... (ríe), no sé cómo decirte..., a mí me sonaba a una mezcla entre aragonés-catalán... No me sonaba..., no me sonaba a algo mío (ríe)... No (ríe). Como curioso pues bien, dices eh... Pero como curiosidad. No sé cómo decirte... no me entra más adentro.

Por otra parte, se distingue el discurso de las personas con procedencia altoaragonesa pero que no han sido herederos de la lengua, los cuales suponen el grupo mayoritario. Estos, muestran simpatía hacia la lengua y una actitud favorable a su difusión en los medios de comunicación, y además valoran de forma positiva la emisión de los escasos programas de la televisión autonómica relacionados con la lengua propia, los cuales manifiestan ver asiduamente. Resulta curioso comprobar que estos sujetos parecen no haber hecho suya la imagen estereotipada del hablante de aragonés que se ha difundido entre los mass media a lo largo del siglo Xx e incluso en nuestros días, sino que más bien cuentan con un sentimiento afectivo hacia la lengua que les lleva a entenderla como una parte de su cultura y su pasado, digna de cierto reconocimiento. Es, quizá, ese contacto parcial pero empírico con la lengua a través de sus antepasados, el que sirve de barrera a la identificación de la misma con la falsa imagen estereotipada del aragonés difundida desde el baturrismo. 
S58: te irías acostumbrando, esto es como todo, cuando te acostumbras y vas oyendo hablar también te vas aficionando y te va gustando [...] la tele es una manera de aprender el idioma, y es más, hay un programa, no sé cuál era..., Bien Dicho, y ya lo hemos visto aquí y van por los pueblos y esto... Y el aragonés salía allí y lo veíamos, porque..., claro, el aragonés no sé si es como el fovano o es muy parecido algunas palabras. No sé si es igual, yo no sé si el aragonés es el fovano..., y pues sí, nos chocan palabras que en fovano eran igual.

¿Consideráis que tendría salida un programa de la tele integro en aragonés?

S3: ¡eso sería lo mejor! O los dibujos de los críos... Porque las expresiones esas a los niños les gustan y las imitan, las hacen así...

S7: sí, eso sería lo mejor.

S1: sí, y es que la gente yo creo que los ve. Ese programa, que ahora no me acuerdo cómo se llama, que es una chica que va por allí aprendiendo... Bien Dicho puede ser, sí. Las noticias..., hombre..., yo veo más bien la versión lúdica y cultural...

S3: hombre, pero el apartado cultural de las noticias..., sí, o el tiempo, que te acostumbres a oír ese vocabulario...

Sin embargo, entre este sector de la población, el que cuenta con raíces altoaragonesas, los sujetos más informados, una minoría de personas que tienen algún tipo de vínculo con la enseñanza del aragonés o con los círculos activistas, muestran un discurso más sólido desde el cual se pone en cuestión la imagen del hablante de aragonés y de la lengua per se que se difunde desde la televisión autonómica:

(Respecto a los que consideran la expresión "co» como muestra del aragonés, de forma errónea) S33: eso es de Zaragoza, ¡eso es de Zaragón! Eso es una barbaridad y es que encima la gente se ríe y la gente lo coge como imitación y vamos jes que es increíble! Y la culpa la tiene el «oregonés» ese de las narices, ¿eh? Mucha, mucha, mucha, mucha culpa, porque esos a lo mejor, si en la TV quisieran es que hablaríamos todos aragonés. ¡Hombre, claro! Pero es que en el programa de Oregón Televisión se ríen del «co» y todo el día «co, co, co, co»... ¡Por favor!

S22: los medios de comunicación y la televisión son un ámbito de actuación urgente, pero no haciendo programas confusos que sí, que en el mejor de los casos pueden despertar algún tipo de curiosidad en mucha gente que los vea...; pero yo principalmente hablo de programas dirigidos a la gente joven y especialmente a los niños. Lo que hace falta de verdad son dibujos animados en aragonés. Yo no le veo mucho sentido a una televisión autonómica si no es, al menos, capaz de promocionar las dos lenguas más minoritarias de Aragón.

A este perfil favorable a la presencia del aragonés en los medios pero crítico al mismo tiempo respecto a la situación actual, se suman los altoaragoneses no hablantes de aragonés y con origen en un territorio estatal plurilingüe (principalmente catalanófono y vascófono): 
S65: [La presencia en los medios] puede influir para gente que no lo conozca, o que, o que... o a los niños en ciertas edades que se dejan influir mucho por el grupo, por ejemplo los adolescentes. Eso sí que en esa época estás muy influido por lo que sale en la tele... (ríe). ¿En la tele? no vas a oír aragonés más que por el programa este de broma [...] una de las cosas que han hecho tanto en País Vasco como en Catalunya ha sido hacer una cadena exclusiva pues que se habla en su idioma. Entonces, ¿series infantiles?, todas traducidas, ¿películas?, traducidas. O sea, traducidas, no, dobladas, que es lo importante. Que te entre... Porque al ver la tele por lo visto utilizas varios sentidos: los de la vista y el oído y entonces lo percibes mejor, lo aprendes más.

S24: Es que el aragonés... Primero que la radio aquí casi no llega, que solo llega la SER y Radio Sobrarbe en algunos puntos pero no en todos. Tele Aragón pues la verdad es que no lo vemos mucho porque es que cada vez que la pongo están haciendo jotas o cosas así (ríe), entonces no la solemos poner mucho. No hay programación infantil, que eso hace mucho. En TV3 les metieron programación infantil y bueno... ¡Es que mucha gente entiende el catalán porque se veía la TV3! ¡La Bola de Drac...! (ríe). ¿Ves? Es que los medios de comunicación, ¡y además programación infantil!, eso, buah, haría mucho, eso haría mucho. Pero luego volvemos a lo mismo de siempre: si a nivel administrativo, institucional, no hay ningún tipo de apoyo ni de interés, qué quieres que te diga...

Para finalizar, encontramos el discurso de los habitantes con procedencia de otros territorios aragoneses y/o del Estado monolingües. En las valoraciones de este grupo, que cuenta con un peso considerable, se hace patente la presencia del prejuicio, el tópico y, a consecuencia de ello, incluso el rechazo hacia la difusión del aragonés. Su discurso muestra una visión fragmentaria de la lengua y, además, hace patente que la imagen baturra difundida en los medios durante el siglo Xx respecto al habitante del Aragón rural ha calado intensamente en esta parte de la población. Extrapolan esta imagen al ámbito lingüístico y es evidente su identificación de la lengua aragonesa con el estereotipo del «tonto listo», del campesino rudo, palurdo e iletrado difundido por el baturrismo:

(Respecto al desarrollo de unos medios de comunicación en aragonés normativo): S24: hoy en día yo creo que eso sería negativo incluso para lo que es la identidad aragonesa porque...

S27: ...porque generaría disputas porque unos querrían el belsetán, otros querrían el chistabín... Yo no lo veo. Para generar disputas entre que habla el belsetán y el que habla «X»... ¡Y a dividir más! ¡Hala, va! (ríe).

S24: en cada valle... Es más, aquí, Gistaín y Plan están a cuatro km. y el chistabín que yo he oído en Gistaín es distinto de que hablan..., que ves bastantes diferencias ya dentro. Vale. Y es evidente que el aragonés que se habla aquí no tiene nada o tiene muy poco que ver con el aragonés de Ansó o de Echo, ¿vale? ¿Cómo vas a unificar algo que pasa por una lengua que no es la lengua materna de nadie? ¡De absolutamente nadie!... 
S14: y luego yo creo que también es que se vendió durante muchos años la idea del de pueblo garrulo y entonces eso también se intentó un poco eliminar. Y yo creo que sí, que todavía queda un poco, en según qué sitios, sí...

S18: ha sido un poco también despreciado por los propios lugareños porque era malsonante o no era de cultos...) también da un poco de miedo el..., como pueden tener similitudes, ¿no?, el castellano y el aragonés, el que saquen mezclas raras, yo creo que a los padres que somos de fuera también nos da un poco de miedo eso. Decimos: 'a ver qué va a decir mi niño, que me va a mezclar, que a ver...', que si esto es castellano y esto es aragonés, a ver qué mezcla hacen... Eso es lo que más miedo también da... Si van a mezclarlo todo. Y eso sí que da mieditis, ¿eh? Porque yo qué sé, dices: ‘¡a ver qué va a hablar la criatura!' (ríe). Entonces...

Pero, además, entre los prejuicios lingüísticos que muestra este sector de la población aparecen muchos otros de los que d'Andrés (2002) determina como propios de la ideología de tipo denigratorio respecto a la lengua minoritaria, destacando, por ejemplo, los de tipo:

- estético («es una lengua que suena mal»):

¿Y vuestra reacción cuál ha sido cuando habéis escuchado esas palabras y expresiones...?

S42: Curiosa, risa. Lo primero que te sale es la risa, ¿no? Y es curioso algunas expresiones, ¿no? Suena... Es una lengua que suena rara, ¿no? Que por eso creo que se ha abandonado, porque se tachaba como una lengua un poco de incultos o que se hablaba mal, ¿no? Yo es que claro, tampoco he profundizado, hay ciertos palabros que sí que es eso, que son un poco malsonantes pero bueno, que hay que respetarlo...

- funcional («no vale para hablar de determinadas cuestiones»):

S60: igual se puede hablar yo qué sé..., de fauna y flora utilizando el aragonés... Que es que tampoco...

S61: a ver, que a mí me parece que si estamos hablando de lo tradicional me parece que encaja...

- cultural («es reflejo de una cultura rural y limitada»):

S35: mi opinión es, para mí el aragonés entraría como una lengua folklórica, ¿`sabes?, que está más para el folklore, como la cultura de un pueblo, la identidad de un pueblo, que se puede estudiar aparte. Pero ni obligar ni entrar como una asignatura [en la escuela]. ¿Por qué? Porque, por eso mismo, porque lo veo como un tema folklórico, específico de Aragón, ¿entiendes? 
- dialectal («no existe como una lengua sino como diferentes hablas»): S49: no hay un aragonés, es lo que tú estás diciendo, son diferentes dialectos y dependiendo del valle cambia totalmente pero es que además hay una disparidad... (...) Yo no lo sé, a mí me han dicho que están haciendo dijéramos que el más extendido y enriqueciéndolo con palabras de uno y de otro y yo digo: «no, se lo están inventando entonces, que es lo mismo que hicieron en Catalunya y en el País Vasco e incluso en Galicia...

- de uso social («nadie habla esa lengua»):

S48: lo están forzando, hablar una lengua muerta, y están gastando el dinero de todos los españoles en que en Aragón se empiece a hablar una lengua que está muerta. Yo eso no lo puedo entender.

\section{Conclusiones}

Los resultados muestran una actitud mayoritariamente neutra-favorable a la presencia de la lengua propia en los medios, especialmente en los territorios donde todavía se conserva con cierto grado de vitalidad, lugares donde, sin embargo, no tienen repercusión las iniciativas desarrolladas desde el ámbito neohablante, lo cual pone de manifiesto la fractura existente entre la comunidad nativa y los nuevos hablantes de aragonés. Esta situación es acorde a los datos obtenidos desde una perspectiva cuantitativa por Campos (2017), según los cuales el 26,4\% de la población altoaragonesa confirma el deseo de poder ver programas de televisión o escuchar la radio en aragonés, un $42 \%$ tiene una actitud neutral (opción «me da igual») al respecto y un 30,7\% manifiesta no tener interés por esta oferta.

Por otra parte, en términos generales, destaca la carencia de información entre la sociedad altoaragonesa respecto a la lengua propia, siendo relevante sobre todo la falta de modelos en la vida pública e intelectual con los que identificar al hablante de aragonés. De este modo, en muchas ocasiones emerge una identificación con modelos tintados por el prejuicio, tópico y/o folklorismo como son las identidades baturras, las cuales se han difundido de un modo recurrente a través de los medios de comunicación y del cine, sobre todo a lo largo del siglo Xx. Esta identificación se encuentra principalmente entre los habitantes de procedencia no altoaragonesa y está relacionada con la presencia de otros prejuicios lingüísticos y una ideología denigratoria (d'Andrés, 2002) respecto a la lengua minoritaria y minorizada. 
Ante esta situación resulta obvia la relevancia de las instituciones en el desarrollo de un tratamiento suficiente y adecuado del aragonés en los medios de comunicación públicos; un tratamiento deseado por la mayor parte de la población altoaragonesa y que puede ser clave para la desaparición de los prejuicios lingüísticos existentes tanto entre los hablantes de la lengua como entre la mayoría de habitantes castellanohablante. Asimismo, hemos de recordar que el interés que la mayor parte de la población altoaragonesa muestra hacia la programación televisiva centrada en la lengua propia, puede resultar un arma de doble filo ya que, si estos contenidos ofrecen una imagen sesgada del aragonés, tal y como algunos informantes denuncian respecto al programa Bien Dicho que se emite en la actualidad, la audiencia receptora puede interiorizar prejuicios lingüísticos como, por ejemplo, una concepción fragmentaria del aragonés, como un conjunto de diferentes dialectos sin una base común y no susceptibles de pasar por un proceso de estandarización y normativización. En este sentido, no debemos olvidar la relevancia que autores como Munné (1980) han aportado a los medios de comunicación respecto al desarrollo de las actitudes hacia las lenguas minoritarias, relevancia corroborada por estudios como el de Baker (1992). De este modo, y ante los datos obtenidos, consideramos urgente la dotación de unos contenidos en y sobre el aragonés en los medios de comunicación autonómicos, los cuales cuenten, además, con cierta función pedagógica que permita tanto terminar con la ruptura que existe entre las diferentes redes de hablantes del aragonés, así como eliminar los prejuicios de la sociedad altoaragonesa derivados de la falta de información sobre la lengua propia y su devenir histórico, tanto desde el punto de vista lingüístico como sociolingüístico.

\section{REFERENCIAS BIBLIOGRÁFICAS}

Alén Garabato, Carmen, 2013, «O occitano na França: qual e para qué usos?» En: Quem fala a minha língua. Santiago de Compostela, Através: 92-129.

Aliaga Jiménez, José Luis, 2012, Las lenguas de Aragón en el primer tercio del siglo veinte. Vol. 1. Inéditos, rarezas y caras B. Zaragoza, Gara d'Edizions y Prensas de la Universidad de Zaragoza.

ANDRÉs, Ramón d', 2002, «La llingua asturiana na sociedá.» En Informe sobre la llingua asturiana. Oviedo, Academia de la Llingua Asturiana: 77-103.

BAKer, Colin, 1992, Attitudes and language. Clevedon, Multilingual Matters.

Bourdieu, Pierre, 2008, ¿Qué significa hablar? Economía de los intercambios lingüísticos. Madrid, Akal. 
CAMPos, Iris Orosia, 2017, Lengua minorizada y enseñanza. Actitudes, metodologías y resultados de aprendizaje en el caso del aragonés. Zaragoza, Prensas de la Universidad de Zaragoza y Gobierno de Aragón. [En prensa].

Ciprés PAlacín, María Ángeles., 2009, «La poesía contemporánea en lengua aragonesa: análisis de su trayectoria y estudio de algunas constantes», Luenga $\mathcal{E}$ fablas, 12-13: 7-52.

Cortes de Aragón, 1997, Dictamen de la Comisión especial de política lingüística. Zaragoza: $B O C A, 105$.

Eito MATEO, Antón, 2013, «El aragonés en la radio, la televisión e Internet.» En José Ignacio López Susín, coord., El aragonés en el siglo XXI. Informe. Zaragoza, Fundación Gaspar Torrente: 73-75.

Enguita Utrilla, José María, 2014, «Actitudes lingüísticas en Aragón.» En María Antonia Martín Zorraquino, coord., Filología, gramática, discurso. Artículos escogidos (1976-2013). Zaragoza: Institución Fernando el Católico: 133174.

GARCÉS SANAGUSTíN, Ángel, «La legislación aragonesa reguladora de sus lenguas: una emersión frustrada», Revista catalana de dret públic, 50: 184-208.

García GuATAs, Manuel, 1999, «La imagen costumbrista de Aragón.» En Localismo, costumbrismo y literatura popular en Aragón. V Curso sobre Lengua y Literatura en Aragón. Zaragoza, Institución Fernando el Católico: 119-123.

Gracia Trell, Alberto, ed., 2014, Somontano en alto. Escritos (1946-1959) e inéditos de Pedro Arnal Cavero. Zaragoza, Aladrada.

Glaser, Barney G. y Anselm L. Strauss, 1967, The discovery of Grounded Theory: strategies for qualitative research. New York, Aldine.

Guillén PARDOS, Enrique, 2012, «El baturro y la identidad aragonesa». [Disponible en: http://www.aragondigital.es/noticia.asp?notid=100161\&secid=21. Acceso el 12/08/2017].

Justicia DE ARAgÓN, 1993, «Informe y Resolución del Justicia de Aragón sobre las lenguas minoritarias de Aragón», Zaragoza: BOCA, 66.

Llera Ramo, Francisco, dir., 2001, Estudio sociolingüístico de las hablas del Altoaragón [inédito].

López Susín, José Ignacio, 2012, Mapa Lingüistico de Aragón (Según el Anteproyecto de Ley de Lenguas de 2001). Zaragoza, Aladrada.

López Susín, José Ignacio, coord., 2012b, El aragonés, una lengua románica. Zaragoza, Rolde de Estudios Aragoneses y Consello d'a Fabla Aragonesa.

López Susín, José Ignacio, 2015, «A lai 3/2013, de 9 de mayo, u a infraproteuzión churidica de as luengas minoritarias d'Aragón», Revista de Llengua i Dret, 63: 186-199.

López Susín, José Ignacio y José Luis Soro Domingo, 2010, Estatuto jurídico de las lenguas propias de Aragón. La Ley 10/2009, de 22 de diciembre. Zaragoza, El Justicia de Aragón. 
Moseley, Chistopher, ed., 2010, Atlas of the World's Languages in Danger. Paris, UNESCO Publishing.

Munné Matamala, Federico, 1980, Psicología Social. Barcelona, CEAC.

Nagore Laín, Francho, 2008, «La política lingüística en la Comunidad Autónoma de Aragón en la década de 1996-2006.» En Max Doppelbauer y Peter Cichon, eds., La España multilingüe. Lenguas y políticas lingüisticas de España. Viena, Praesens Verlag: 131-167.

Postlep, Sebastian, 2012, «'Este per no ye d'a mía tierra’: Percepción 'científica’ y percepción 'inexperta' del contínuum dialectal altoaragonés», Alazet, 24: 77116.

RAmos, Rubén, 2014, «L'aragonés y os media... ¿Una historia d'amor imposible?» En Chabier Gimeno y Natxo Sorolla, coords., Actas de las II Jornadas Aragonesas de Sociología. Grupo de trabajo 'Lenguas e Identidades'. Zaragoza, Institución Fernando el Católico, Prensas de la Universidad de Zaragoza y Gara d'Edizions: 121-137.

Satué Sanromán, José María, 2016, «O zaguer día», Heraldo de Aragón (28 de agosto de 2016): 55.

Serrano Lacarra, Carlos, 2014, País y lenguas. La lengua aragonesa en la revista El Ebro (1917-1936). Zaragoza, Aladrada.

UNESCO, 1996, «Universal declaration on linguistic rights». [Disponible en: http:// www.unesco.org/cpp/uk/declarations/linguistic.pdf, acceso el 12/08/2017]. 